\title{
The Behavior of the Hydrogen Atom under Different Potential Well
}

\author{
Wei-Xing Xu \\ Newtech Monitoring Inc., Oshawa, Ontario, Canada \\ Email: xweixing@hotmail.com
}

How to cite this paper: Xu, W.-X. (2019) The Behavior of the Hydrogen Atom under Different Potential Well. Optics and Photonics Journal, 9, 60-73. https://doi.org/10.4236/opj.2019.95007

Received: April 19, 2019

Accepted: May 19, 2019

Published: May 22, 2019

Copyright () 2019 by author(s) and Scientific Research Publishing Inc. This work is licensed under the Creative Commons Attribution International License (CC BY 4.0).

http://creativecommons.org/licenses/by/4.0/

\begin{abstract}
In this work we studied the behavior of hydrogen atom under different potential well and found that with decreasing the distance between the electron and proton, the more multiple interaction terms start to take effect and the potential well becomes more and more sensitive to the distance between the electron and proton. Based on these results, the potential well for the neutron decaying is determined. Furthermore, the general topics, such as the origin of nuclear energy, the fundamental forces in the nature and the matter with negative mass, also are discussed. At the end of this work, the duality model of our universe is put forth.
\end{abstract}

\section{Keywords}

Neutron Decaying, Multiple Interactions, The Negative Mass,

The Handedness, The Gravity, The Fundamental Forces

\section{Introduction}

Since the human being appeared, the activity for the human being to understand and utilize the nature is never stopped. In the long river of history, the human being made a series of brilliant achievements and great discoveries. These important progresses encourage the human being continuously moving forward in exploring the secret of nature.

On the road for the human being to explore the nature, we can cite a few milestones, such as, in mathematics, the pioneer people set up the integration/differentiation [1] and different geometries [2] [3]; in physics, they discovered the gravity and set up the Newton mechanics [4], the special and general relativities [5] and quantum mechanics [6]. Later on, the science is divided into different branches and more the details of the nature have been revealed. When we look back the whole history of science, we are surprised and at the same time, 
proud of the achievements made by the human being in the past.

Now most of people live in this scientific building established by the pioneer scientists, they are satisfied and feel comfortable. But for us, we are not so confident in this situation and always worry about whether the basis of science is founded on the solid ground or not. Therefore we try to use the magnifier to find out any tiny mistake or defect in the present scientific building, which may cause the whole building collapse in the future.

As a tiny step in this effort, we will take the hydrogen atom as an example to start our discussion.

\section{Theoretical Consideration}

It is no doubt that the simplest system under consideration of scientific world is the hydrogen atom, which is the only system we can get the analytical solution of the Schroedinger Equation [7]. The corresponding Hamilton operator is

$$
H=-\frac{1}{2}\left(\frac{\partial^{2}}{\partial r^{2}}+\frac{2 \partial}{r \partial r}\right)-\frac{1}{r}
$$

In general cases, the solution of Schroedinger Equation (1) can give satisfied explanation about the experimental result. However, if we consider some special cases, the conventional Hamilton operator for the hydrogen atom is not good enough [8] [9] [10] [11] [12]. Therefore, in this work, we will consider the more general form of hydrogen Hamilton operator (as a first order approximation, we will not include the relativity effect).

$$
H=-\frac{1}{2}\left(\frac{\partial^{2}}{\partial r^{2}}+\frac{2 \partial}{r \partial r}\right)-\sum_{n=1}^{\infty} \frac{A_{n}}{r^{n}}
$$

where $A_{n}$ is a constant.

Using $\phi_{1 s}$ to start our discussion, then,

$$
\begin{aligned}
\int_{0}^{\infty} \varphi_{1 s} H \varphi_{1 s} \mathrm{~d} \tau & =\int_{0}^{\infty} \frac{1}{\sqrt{\pi}} \mathrm{e}^{-r}\left[-\frac{1}{2}\left(\frac{\partial^{2}}{\partial r^{2}}+\frac{2 \partial}{r \partial r}\right)-\sum_{n=1}^{\infty} \frac{A_{n}}{r^{n}}\right] \frac{1}{\sqrt{\pi}} \mathrm{e}^{-r} \mathrm{~d} \tau \\
& =\frac{1}{2}+I_{3}
\end{aligned}
$$

where

$$
I_{3}=-\int_{0}^{\infty} 4 \sum_{n=1}^{\infty} \frac{A_{n}}{r^{n-2}} \mathrm{e}^{-2 r} \mathrm{~d} r=\sum_{n=1}^{\infty} I_{3 n}
$$

For $n=1, A_{1}=1$,

$$
I_{31}=-\int_{0}^{\infty} 4 r \mathrm{e}^{-2 r} \mathrm{~d} r=-1
$$

Then the total energy of hydrogen atom is $E=-1 / 2$. This case is just the result described by the conventional Hamilton operator of the hydrogen atom.

For $n=2$, then, we have,

$$
I_{32}=-\int_{0}^{\infty} 4 \frac{A_{2}}{r^{2-2}} \mathrm{e}^{-2 r} \mathrm{~d} r=-\int_{0}^{\infty} 4 A_{2} \mathrm{e}^{-2 r} \mathrm{~d} r=-2 A_{2}
$$


This will make the total energy of the hydrogen atom, $E=-1 / 2-2 A_{2}$.

From the expression of $I_{32}$, we know that interaction force is inversely proportional to the cubic of the distance between the electron and proton. At moment, we don't know whether there exists other origin of the interaction force inversely proportional to the cubic of the distance or not. But we do know one origin of the interaction force inversely proportional to the cubic of distance between the electron and proton, that is, if the electron and proton are not geometry point charge (the geometry point charge means the electron and proton have no volumes [13]), then interaction force inversely proportional to the cubic of distance between the electron and proton does exist. To my knowledge, some researchers declare the electron doesn't have volume, but the proton does have. Therefore, we can reasonably think the interaction force inversely depending on the cubic of distance reflects the real situation in the interaction between the electron and proton. Just in normal condition, this interaction force is too small to be counted.

For $n=3$, we have,

$$
\begin{aligned}
I_{33} & =-\int_{0}^{\infty} 4 \frac{A_{3}}{r^{3-2}} \mathrm{e}^{-2 r} \mathrm{~d} r \\
& =-4 \int_{0}^{\infty} \frac{A_{3}}{r} \mathrm{e}^{-2 r} \mathrm{~d} r \\
& =-4 A_{3}\left[\lg r+\frac{(-2)}{1 !} r+\frac{(-2)^{2}}{2 \cdot 2 !} r^{2}+\frac{(-2)^{3}}{3 \cdot 3 !} r^{3}+\cdots\right]_{\delta}^{\infty}
\end{aligned}
$$

where $\delta$ is a smallness. For convenience, we can define $Q(r)$ as

$$
Q(r)=\left[\lg r+\frac{(-2)}{1 !} r+\frac{(-2)^{2}}{2 \cdot 2 !} r^{2}+\frac{(-2)^{3}}{3 \cdot 3 !} r^{3}+\cdots\right]_{\delta}^{\infty}
$$

then,

$$
I_{33}=-4 A_{3} Q(r)
$$

In the range of $a_{0} \sim r_{m}$ (here $a_{0}$ is the Bohr radius; $r_{m}$ is the neutron radius), $Q(r)$ is positive, therefore, $I_{33}$ term makes total energy, $\mathrm{E}$, become more negative. We may ask what the physical basis for the $I_{33}$ ? From the expression of $A_{3} / r^{3}$, we know that the dipole-dipole interaction potential energy has the form $1 / r^{3}$, this gives us a hint that $I_{33}$ comes from the dipole-dipole interaction between the electron and proton [14]. From the expressions of Equations (8) and (9), we know that this interaction changes with the $r$, the closer the electron to the proton, the stronger the dipole-dipole interaction becomes.

For $n=4$, we have,

$$
\begin{aligned}
I_{34} & =-4 \int_{0}^{\infty} \frac{A_{4}}{r^{4-2}} \mathrm{e}^{-2 r} \mathrm{~d} r \\
& =-4 \int_{0}^{\infty} \frac{A_{4}}{r^{2}} \mathrm{e}^{-2 r} \mathrm{~d} r \\
& =-4 A_{4}\left[-\frac{\mathrm{e}^{-2 r}}{r}-Q(r)\right]_{\delta}^{\infty} \\
& =\left.4 A_{4} \frac{\mathrm{e}^{-2 r}}{r}\right|_{\delta} ^{\infty}+\left.4 A_{4} Q(r)\right|_{\delta} ^{\infty}
\end{aligned}
$$


From the discussion above, we know that the second term is positive and the first term is negative in the range of $a_{0} \sim r_{m}$. The second term will be cancelled by the $I_{33}$ (at least partially cancelled). Therefore, the net contribution of $I_{34}$ to the total energy, $E$, is negative, which means that $I_{34}$ will make the electron binding to the proton stronger than that in hydrogen atom under normal case.

To our knowledge, the dipole-quadruple interaction energy is proportional to $1 / I^{4}$ [15]. If this is the unique origin of $I_{34}$, then we are sure there is the interaction potential energy between the electron and proton which is proportional to $1 / r^{4}$.

For $n=5$, we have,

$$
\begin{aligned}
I_{35} & =-4 \int_{0}^{\infty} \frac{A_{5}}{r^{5-2}} \mathrm{e}^{-2 r} \mathrm{~d} r \\
& =-4 \int_{0}^{\infty} \frac{A_{5}}{r^{3}} \mathrm{e}^{-2 r} \mathrm{~d} r \\
& =\left.2 A_{5} \frac{\mathrm{e}^{-2 r}}{r^{2}}\right|_{\delta} ^{\infty}-\left.4 A_{5} \frac{\mathrm{e}^{-2 r}}{r}\right|_{\delta} ^{\infty}+\left.8 A_{5} Q(r)\right|_{\delta} ^{\infty}
\end{aligned}
$$

Compared to the dipole-quadruple interaction, the $I_{35}$ has term proportional to $1 / r^{2}$. This means that the interaction energy becomes more and more sensitive to the distance between the electron and proton. One may wonder what kind of interaction potential between the electron and proton will depend on $1 / r^{5}$. From the basic electromagnetic theory, we know that one origin is the quadruple-quadruple interaction potential between the electron and proton, which is proportional to $1 / r^{5}$. Not like the dipole-quadruple interaction, the dependence of the quadruple-quadruple interaction on $r$ becomes much more complicated. It can be seen that in the expression of $I_{35}$, some term will make the total energy of hydrogen atom, $E$, increasing, some term will make the total energy decreasing. But the net influence of $I_{35}$ on $E$ will be determined by the leading term, that is, $\left.A_{5} \frac{\mathrm{e}^{-2 r}}{r^{2}}\right|_{\delta} ^{\infty}$. This term keeps negative. Therefore, the net effect of $I_{35}$ on $E$ is to make $E$ increasing. Compared $I_{33}, I_{34}$ and $I_{35}$, it is noticed that except the leading term, there exists cancellation effect among the other terms. This fact makes us a little easy to simplify our following discussion.

Following the above logical line from $I_{31}, I_{32}, I_{33}, I_{34}$ and $I_{35}$, we can reasonably deduce that with increasing more and more multiple interaction between the electron and proton, such as the point-point charge interaction, point-dipole interaction, dipole-dipole interaction, dipole-quadruple interaction, quadruple-quadruple interaction, quadruple-octupole interaction, octupole-octupole interaction..., the dependence of the interaction energy on $r$ will become more and more sensitive and steeply. Following the same way, we can get the general form for $I_{3 n}$ as,

$$
I_{3 n}=-\left.\frac{4 A_{n}}{1-m} \frac{\mathrm{e}^{-2 r}}{r^{m-1}}\right|_{\delta} ^{\infty}+\frac{8 A_{n}}{m-1} \int_{\delta}^{\infty} \frac{\mathrm{e}^{-2 r}}{r^{m-1}} \mathrm{~d} r
$$


where $m=n-2$.

$I_{3 n}$ includes a lot of terms and becomes much more complicated. Fortunately, as we discussed above, $I_{3 n}$ is mainly determined by the leading term and there exists cancellation among the other terms. Therefore, in most cases, we can study the behaviors of hydrogen atom based on the leading term in $I_{3 n}$.

Here we can take the conversion from the neutron to hydrogen atom as an example to demonstrate how to apply the concept developed above in practice.

Experimentally, the half life of neutron decaying is determined to be $611 \mathrm{~s}$ [17]. Supposing the neutron decaying obeys the first order kinetic reaction [18], then,

$$
\begin{gathered}
t_{1 / 2}=\frac{\ln 2}{K(T)} \\
K(T)=\frac{k_{b}}{h} \exp \left(\frac{-\Delta G^{\#}}{k_{b} T}\right)
\end{gathered}
$$

where $K(T)$ is the rate constant of neutron decaying; $k_{b}$ is the Boltzmann's constant; $h$ is the Planck constant; $T$ is the absolute temperature; $\Delta G^{\#}$ is the free energy for the neutron decaying.

Setting the temperature at $298 \mathrm{~K}$ in our calculation, then

$$
\Delta G^{\#}=0.034206333 \text { (a.u.) }
$$

From the Equation (3), we know that the potential energy for the neutron to be stable is,

$$
\int_{\delta}^{\infty}-4 \frac{A_{n}}{r^{n-2}} \mathrm{e}^{-2 \alpha r} \mathrm{~d} r
$$

where $\alpha$ is the compressed factor as we defined in previous work [19].

Based on our previous result, in order to bring the electron close enough to the proton, the total energy of hydrogen atom will be [19]

$$
E=\frac{1}{2} \alpha^{2}-\alpha
$$

and the

$$
\langle r\rangle=\frac{3}{2 \alpha}
$$

where $\alpha$ is compressing factor defined in our previous work and $\langle r\rangle$ is the expectation distance between electron and proton [19].

Now we get

$$
\int_{\delta}^{\infty}-4 \frac{A_{n}}{r^{n-2}} \mathrm{e}^{-2 \alpha r} \mathrm{~d} r+\frac{1}{2} \frac{9}{4\langle r\rangle^{2}}-\frac{3}{2\langle r\rangle}=\Delta G^{\#}
$$

Supposing the $r$ and $\langle r\rangle$ are the same and replacing $\delta$ by $r$, then, the energy of the system should have a minimum corresponding the neutron radius, $r_{m}$. Therefore, we can differentiate Equation (18), we get,

$$
\frac{4 A_{n}}{r_{m}^{n-2}} \mathrm{e}^{-2 \alpha r_{m}}-\frac{9}{4 r_{m}^{3}}+\frac{3}{2 r_{m}^{2}}=0
$$


Replacing the $\alpha$ in equations (18) and (19), we get,

$$
\begin{gathered}
\frac{-1}{n-1}\left[\frac{4 A_{n}}{r_{m}^{n-2} \mathrm{e}^{3}}\right]+\frac{9}{8 r_{m}^{3}}-\frac{3}{2 r_{m}^{2}}=\frac{\Delta G^{\#}}{r_{m}} \\
\frac{4 A_{n}}{r_{m}^{n-2} \mathrm{e}^{3}}-\frac{9}{4 r_{m}^{3}}+\frac{3}{2 r_{m}^{2}}=0
\end{gathered}
$$

Combining the equations (20) and (21), we determined the two parameters, $A_{n}$ and $n$ are

$n=3$ and $A_{n}=4.94 \times 10^{10}$. Here we get the potential form for the neutron decaying as

$$
P=-\frac{4.94 \times 10^{10}}{r^{3}} \text { (a.u.) }
$$

This form is different from those expressions for the nuclear potential proposed in the literature [20]. This means that a proton combining an electron to form a neutron only involved the dipole-dipole interaction and potential well is not so deep, therefore, the neutron is not so stable at the normal condition.

\section{Discussions}

Based on the result above, we like to discuss some interesting issues below.

\subsection{When the Neutron Decays, a Huge Amount of Energy Is Released. Where the Huge Energy Come from?}

As we know, in order for the dipole-dipole like force to take effects to form the neutron, the electron must be close enough to the proton. In our previous work, we demonstrate that to push the electron close to the proton, we have to do work to push the electron close enough to the proton, that means the energy of system will increase, which can be calculated from the equations developed in our previous work, (see Equation (16) and Equation (17) above). That is,

$$
E=4.92 \times 10^{9} \text { (a.u.) }
$$

Here we take the $\langle r\rangle$ equal to the radius of the neutron as approximation in calculation. This is a huge amount of energy compared to the total energy at the normal condition. Therefore, corresponding to the neutron decaying, this huge amount of energy will be released in the form of radiation and kinetic energies of the proton and electron. Even though we use some approximations in our calculation, such as we do not include the relativity effect, but our result gives a reasonable explanation about the origin of the huge amount of energy during the neutron decaying, that is, the energy released in the process of the neutron decaying is the compressed electromagnetic energy, not others.

\subsection{Does There Exists the Different Excited States of Neutron?}

In above discussion, the result is based on the fact that we neglect the other term in the expression of $I_{33}$. If the left terms cannot completely cancel out, then, it 
will create some different energy levels in the potential well formed by the leading term in the expression of $I_{33}$ (in fact, $I_{33}$ can be expressed as $Q(r)$ defined above, and it does show some swallow potential sub-well when $r$ approaches to $\left.r_{m}\right)$. That means the neutron can be excited to different energy levels before it suffers the decaying. If we can develop some techniques to study the behavior of neutron at the different excited states, it will be very interesting.

\subsection{What Will Happen If the Power of $r$ Goes to High?}

Following the same logical line above, we can reasonably deduce that the dipole-quadruple interaction potential will be proportional to $1 / r^{4}$, the quadruple-quadruple interaction potential proportional to $1 / r^{5} \ldots$ It is noted that the power of $r$ increases continuously with the high multiple interaction involved, that means the potential well will become more and more steeply.

Now we are wondering that at the certain stage, the $I_{3 n}$ terms will make the total energy of system becomes zero. This can be realized in two ways, one is to decrease the $r$ by pushing the electron closer to the proton; another way is to increase the power of $r$. In fact, these two effects work cooperatively together. That is, decreasing $r$, then the higher multiple terms will be involved; therefore, the power of $r$ will increase. As a result, the $I_{3 n}$ term will become more negative. This fact tells us that with decreasing $r$ and at the same time increasing the power of $r$, sooner or later, the $I_{3 n}$ term will make the total energy of hydrogen atom become zero. What the physical meaning for this zero energy of system? That means the whole system does not have static mass $\left(E=m c^{2}\right.$, if $\left.m=0, E=0\right)$ [21]. But due to the energy conservation law, the whole system only can exist as the electromagnetic field, it just looks like that the hydrogen atom at this situation be "vaporized" into photon, in which way the system keeps the energy conservation law still valid. At this point, the matter as a physical entity disappeared from the vision of human being. In fact, we can determine the $r$ at which the matter will disappear from the vision of human being experimentally. For example, making the electron collides with the proton, if the collision energy is enough high, the electron-proton will become the electromagnetic field instead of physical particle.

\subsection{What the Situation Will Be If the Energy of System Becomes Negative?}

Beyond the matter be "vaporized" into the electromagnetic wave, if the energy of system continuing decrease (for example, the collision energy is enough high to push the electron very, very close to the proton, then, the potential term in $I_{3 n}$ will make the energy of whole system becomes negative), the total energy of system will become negative [22]. There exist two ways to explain this situation. One is to say the system form a black hole. This is the popular opinion about the system under extremely high pressure. In this case, the electromagnetic wave can't escape from the black hole. That means the spacetime of black hole move faster than the electromagnetic wave. Therefore, the spacetime of black hole al- 
ways covers the electromagnetic wave inside. However, based on our previous work [23], it is impossible for the spacetime moving faster than the electromagnetic wave either.

The second explanation is that based on the Einstein's mass-energy relation, the system can change the extra energy into the negative mass. This negative mass system keeps the gravity in attractive and also takes the speed of the electromagnetic wave as the highest speed. As we discussed in our previous work, if the speed of the electromagnetic wave is beyond the speed of spacetime, the negative mass will appear. This extra energy which makes the speed of the electromagnetic wave beyond the speed of the spacetime, is changed into the negative mass. In this way, the speed of the electromagnetic wave can be kept as the highest speed and constant.

It is interesting that if we consider the interaction between the matter with positive mass and the matter with negative mass, we find that the gravity becomes repulsive [24] [25]. Currently, most scientists believe that our universe is in accelerated expansion. But they do not know what force is responsible for this accelerated expansion. Then they figure out there exist some dark matter and dark energy, therefore, they try to find out the dark matter and dark energy but to my knowledge, till now this search for the dark matter and dark energy is still no result.

From our discussion above, the existence of the matter with negative mass gives a reasonable explanation for the accelerated expansion of our universe, that is, the universe is divided into two parts, one part has the matter with the positive mass, another part has the matter with negative mass. Both parts of our universe keep the speed of the electromagnetic wave as the highest speed and constant. All existing physical laws are valid and keep the same form in both parts of our universe. Between both parts of our universe, only interaction can be realized through the electromagnetic wave and gravity.

Due to the gravity comes from the interaction between the spacetime and the matter or compressed spacetime as we discussed in our previous work, therefore, in both parts of our universe, the gravity interaction between the matter with positive mass and the matter with negative mass is repulsive in character. This may explain why our part of universe is in accelerated expansion but we can't find the origin of the force responsible for this accelerated expansion.

The accelerated expansion of our universe puzzled the researchers long time. Here we offered our explanation. The matters in both parts of universe can't exchange freely because they have to become or be "vaporized" into the electromagnetic wave before they exchange or they have to become the electromagnetic wave before they enter another part of our universe. And due to this condition, we can't "see" the matter in another part of universe because they are beyond our vision.

In our previous work [23], we mentioned that the matter with negative mass may be the antimatter. If this is true, then, we can verify whether the matter with 
negative mass is the antimatter or not by measuring the gravity between the matter and antimatter. If the gravity between the matter and antimatter is repulsive in character, then the matter with negative mass is the antimatter, otherwise the matter with negative mass is something else.

\subsection{The Origin of Our Universe}

Conventionally, most researchers believed that our universe comes from nothing by the quantum fluctuation [26]. The puzzle is if our universe is created from nothing by the quantum fluctuation, then averagely, the total energy of our universe should be zero. But in fact our universe has net energy. Where this net energy comes from? Based on our discussion above, our universe consists of two parts, one part has positive energy and another part has negative energy. Two parts of our universe interact on each other through the electromagnetic field and gravity but total energy of whole universe is zero (Figure 1).

At moment, we still can't definitively know whether the antimatter is the matter with negative mass or not but a hint of the interaction between the matter and antimatter implies that the antimatter is the matter with negative mass because experimentally, we know that when the antimatter meets the matter will annihilate (we prefer to use "vaporize") into the electromagnetic wave.

\subsection{Where the Atomic Energy Comes From?}

Traditionally, we explain the origin of atomic energy based on the Einstein's mass-energy relation, $E=m c^{2}$. Generally, this is true. We can calculate the energy released from the nuclear reaction by counting the difference of mass before and after the reaction, then, using $E=m c^{2}$, the total energy released in reaction is calculated. However, we are not just satisfied with the result of the nuclear energy releasing from the system, but also we hope to know whether the energy released from the nuclear reaction is different kind of energy from the electromagnetic energy and how this huge amount of energy be built in nucleus at the beginning.

From our discussion above and previous work [19], we know that in order for the multiple interaction takes effect, the electron must be close enough to the proton; therefore, the electron orbital must be compressed or contracted. Our previous work tells us that the compressing or contracting the wave function of the electron will cause the increasing the inner energy of system, that is, in

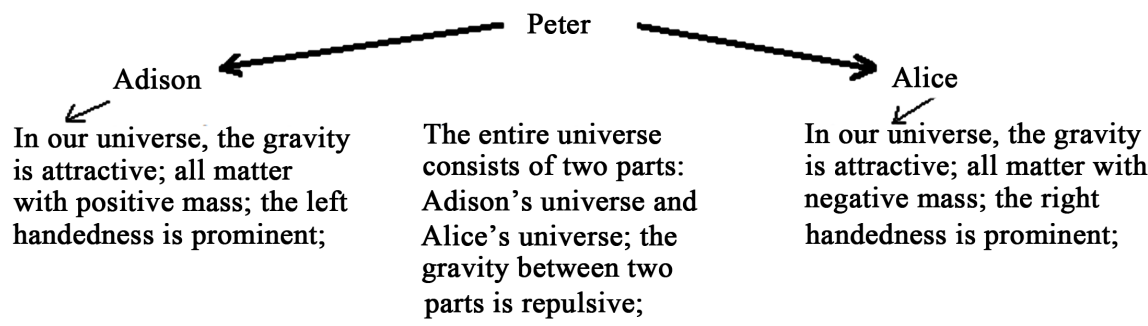

Figure 1. The illustration of the duality model of universe. 
order to make the high multiple interaction to take effect, a huge amount of energy should be input into the system which is stored as compressed electromagnetic energy. Therefore, when fission or fusion reaction occurs, a huge amount of energy gives off from the system in kinetics and radiation energies which comes from this part of stored electromagnetic energy.

It is clear that this form of energy is primarily from the electromagnetic energy. Therefore, the so-called nuclear energy is the electromagnetic energy, not a different form of energy. Our result here also denies the nuclear force as an independent force from the electromagnetic force. In fact, the nuclear force is a compressed electromagnetic force. Based on the same idea, we also can study the interaction between the neutron and proton by observing the behavior of the ionized molecular hydrogen under different potential well.

\subsection{Does the Electron Have Volume?}

In the literature [27], some researchers believe that the electron has no volume. Some theoretical result also reveals that the electron should have no volume. Here, based on the result above, our opinion is that the matter can be beyond our "vision". For example, if we measure the volume of electron by collision, in most time the volume of electron will become smaller and smaller with increasing collision energy. If the collision energy is enough high, the electron will be "vaporized" into the electromagnetic wave, there may exist a tiny possibility for electron being kicked into another part of universe where the electron has negative mass. Experimentally, the antimatter, such as antielectron does be made in this way in the Lab. It doesn't matter the electron be vaporized into the electromagnetic wave or changed into the matter with negative mass, for us, it looks like the electron has no volume.

Even though at moment, our explanation about the volume of electron is not definitive, but to explore the detail mechanism of the conversion between the matter and antimatter or matter with negative mass should be an interesting topic in the future.

\subsection{The Interaction between the Photon and Free Electron}

In our previous work [28], we know that in our part of universe, the interaction of photon with free electron will make the photon lose energy and cause the red frequency shifting. And since the photon has right and left handedeness, the right handedness photon will lose more energy compared to the left handedness photon. That means the electron will get more energy by interacting with the right handedness photon. Therefore, we assign this is the reason (at least, one of the reason) why our part of universe has more the left handedness. However, if the other part of universe consists of negative mass, then the photon will get more energy instead of losing energy by interacting with the electron with negative mass. That means the photon will show blue frequency shifting and the electron with negative mass will lose energy instead of getting energy. Further- 
more, the right handedness photon will get more energy than the left handedness photon in the process of interacting with the electron with negative mass. This result tells us that in other part of universe, it should be prominent in right handedness.

From the discussion above, it is obvious that between both parts of universe it doesn't obey the mirror symmetry but the entire universe keep the energy, handedness constant or zero.

Our work here also provides a way to explore the matter with negative mass by the matter interacting with the handedness photon. Furthermore, based on the concept developed above, we also can verify whether the antimatter is the matter with negative mass or not. Because from the previous work, we know that for changing the sign of electron charge, it only affects the phase of wave function of electron, not the energy. If the antimatter is not the matter with negative mass, then, only the phase of wave function of electron, not the absolute value of energy, will be different from the normal matter situation, otherwise, not only the phase of the wave function of electron but also the absolute value of energy, should be different in both cases.

\subsection{The Fundamental Forces in Nature}

Here we also like to discuss the different interactions in the nature. Basically, it is believed that there exist four fundamental forces, that is, the electromagnetic force, weak force, strong force and gravity [29]. In scientific world, a lot of scientists try their best to unify the four fundamental forces, called unification theory. However, based on our discussion above, all experimental results can be reasonably explained by the electromagnetic interaction except the gravity (as we pointed out in our previous work, the gravity comes from the relative movement between the spacetime and the compressed spacetime, not created by the electromagnetic interaction). We imagine if at the beginning, scientist gives the same name to the weak force, strong force and the electromagnetic force, a lot of scientists may lose their jobs, but good point is the human being can save a huge amount of resources and time for other useful stuff instead of wasting resources and time on proving the four forces really the same stuff, just having different names.

Based on our previous work [23], the gravity comes from the interaction between the spacetime and the matter or compressed spacetime. Therefore, it is really different force from the electromagnetic force. How and why we must unify two different forces together? This is the main reason why most effort to unify the gravity and electromagnetic forces are failed.

Another ridiculer situation is that in order to understand our universe, Euclid developed the geometry to describe the motion and position of matter in our universe, called Euclid's geometry. Riemann's set up another kind of geometry, called Riemann's geometry and many more. It is no doubt that these geometries are the achievements of mathematicians in the past. The problem is the scientist 
uses these geometries to describe our universe. The situation becomes this scientist use this geometry to describe our universe for this problem, that scientist use that geometry to describe our universe for that problem. For example, Einstein use the Riemann's geometry to describe our universe and conclude that the gravity is due to the curved spacetime (we have different opinion on the origin of the gravity, the detail can be found in our previous work [23]). Lobackevskian developed another kind of geometry, called Lobackevskian's geometry [30], to describe our universe and declare that the Lobackevskian's geometry can explain almost all questions regarding our universe, such as four fundamental forces unification and expansion of our universe. It looks like our universe can arbitrarily take its geometry to meet the requirement for different questions. What really geometry of our universe depends on the scientist's will, not fixed. We don't think this is correct situation. Since our universe is only one universe, we can't say today our universe is the Euclid's geometry, but tomorrow say our universe is Riemann's geometry and third day another scientist comes to tell us our universe is other geometry.

Even though our physical picture is approximate because we do not consider the relativity effect but at moment, this is enough for scientific world. The future work will be to include the relativity effect and quantitatively to calculate the interesting physical parameter governing our universe.

\section{Conclusion}

In this work, we set up the potential expression for the neutron decaying and also reveal the fact that there only exist two fundamental forces in the nature, that is, the electromagnetic force and gravity. The duality model of our universe is put forth, on which several puzzles regarding our universe can find reasonable explanation, such as the origin of our universe, the volume of the electron, the accelerating expansion of our universe and the origin of the nuclear energy. Even though at moment our physical picture does not include the relativity effect but generally, our physical picture regarding our universe should be correct. Our work also pointed out how experimentally to test our physical model regarding our universe and how to verify whether the antimatter is the matter with negative mass or not. Future work should go from the qualitative description of our universe to the quantitative description of our universe.

\section{Acknowledgements}

The author would like to say thanks to the clients of the new tech monitoring Inc., it is the clients' loyalty that keeps our business going on smoothly, therefore, the author can spend time to think scientific problem.

\section{Conflicts of Interest}

The author declares no conflicts of interest regarding the publication of this paper. 


\section{References}

[1] Harris, J.W. and Stöcker, H. (1998) Handbook of Mathematics and Computational Science. Springer-Verlag, New York. https://doi.org/10.1007/978-1-4612-5317-4

[2] Hartshorne, R. (2000) Geometry: Euclid and Beyond. Springer-Verlag, New York. https://doi.org/10.1007/978-0-387-22676-7

[3] Eisenhart, L.P. and Veblen, O. (1922) The Riemann Geometry and Its Generalization. Proceedings of the National Academy of Sciences of the United States, 8, 19-23. https://doi.org/10.1073/pnas.8.2.19

[4] Taylor, J.R. (1939) Classical Mechanics. University Science Books, Edwards Brothers, Inc., Lillington.

[5] Einstein, A. (1915) Die Feldgleichungen der Gravitation. In: Sitzungsberichte der Königlich Preußischen Akademie der Wissenschaften, Brandenburgische Akademie, Berlin, 844-847.

[6] Liboff, R.L. (2003) Introductory Quantun Mechanics. Fourth Edition, Addison Wesley, Boston.

[7] Walter, T. and Urban, P.O. (1977) Schroedinger Equation. Springer-Verlag, Berlin. https://doi.org/10.1007/978-3-7091-7673-3

[8] Kaushal, R.S. and Parashar, D. (1992) On the Quantum Bound States for the Potential V $(\mathrm{r})=\mathrm{ar}^{2}+\mathrm{br}^{-4}+\mathrm{cr}^{-6}$. Physics Letters A, 170, 335-338.

https://doi.org/10.1016/0375-9601(92)90882-M

[9] Dong, S.-H., Ma, Z.-Q. and Esposito, G. (1999) Exact Solutions of the Schroedinger Equation with Inverse-Power Potential.

[10] Okon, I.B., Ituen, E.E., Popoola, O. and Antia, A.D. (2013) Analytical Solution of Schroedinger Equation with Mie-Type Potential Using Factorisation Method. International Journal of Recent advances in Physics, 2, 1-7.

[11] Hassanabadi, H., Hamzavi, M., Zarrinkamar, S. and Rajabi, A.A. (2011) Exact Solutions of N-Dimentional Schroedinger Equation for a Potential Containing Coulomb and Quadratic Terms. International Journal of Physical Sciences, 6, 583-586.

[12] Burke, R. and Weatherford, C.A. (2014) Two-Body Dirac Theory. International Journal of Physical Sciences, 4, 797-815. https://doi.org/10.9734/PSI//2014/6994

[13] Carter, J. (2013) My Discoveries and Inventions of the Physics of Mass, Space, Time and Gravity. Absolute Motion Institute, Enumclaw.

[14] Yamamoto, M. (2008) Charge-Charge, Charge-Dipole, Dipole-Charge, Dipole-Dipole Interaction. Dept. of Energy and Hydrocarbon Chemistry, Graduate School of Engineering, Kyoto University, Kyoto.

[15] Davison, W.D. (1968) Atomic Dipole-Quadrupole Dispersion Forces. Journal of Physics B: Atomic and Molecular Physics, 1, 139. https://doi.org/10.1088/0022-3700/1/5/536

[16] Daniel, Z. (2003) CRC Standard Mathematical Tables and Formulae. 31st Edition, Chapman \& Hall/CRC Press, Boca Raton. https://doi.org/10.1201/9781420035346

[17] US National Institute of Standards and Technology (2015) CODATA Value: Proton Mass. The NIST Reference on Constants, Units and Uncertainty.

[18] IUPAC (1997) Compendium of Chemical Terminology. 2nd Edition, Blackwell Scientific, Oxford.

[19] Xu, W.-X. (2018) The Fundamental Mechanism for the Collision/Pressure Induced Optic Effect. Optics and Photonics Journal, 8, 90-97.

[20] Rowlinson, J.S. (1989) The Yukawa Potential. Journal of Physics A: Statistical Me- 
chanics and Its Applications, 156, 15-34.

https://doi.org/10.1016/0378-4371(89)90108-8

[21] Ives, H.E. (1952) Derivation of the Mass-Energy Relation. Journal of the Optical Society of America, 42, 540-543. https://doi.org/10.1364/JOSA.42.000540

[22] Ai, X. (2018) Available Face-Changing Effect. Journal of Modern Physics, 9, 2193-2205. https://doi.org/10.4236/jmp.2018.912138

[23] Xu, W.-X. (2017) Why the Speed of Light Keeps Constant? Optics and Photonics Journal, 7, 67-73.

[24] Bagdoo, R. (2019) Scenario for the Origin of Matter (According to the Theory of Relation). Journal of Modern Physics, 10, 163-175. https://doi.org/10.4236/jmp.2019.102013

[25] Bourdillon, J. (2018) Many-Body-Gravitational Solution for Galactic Rotational Velocities, Including Possible Negative Mass. Journal of Modern Physics, 9, 1304-1316. https://doi.org/10.4236/jmp.2018.96079

[26] Aberg, J. (2018) Fully Quantum Fluctuation Theorems. Physical Review X, 8, Article ID: 011019. https://doi.org/10.1103/PhysRevX.8.011019

[27] Udwadia, F.E. and Kalaba, R.E. (2007) Analytical Dynamics: A New Approach. Cambridge University Press, Cambridge.

[28] Xu, W.-X. (2016) The Interaction between Photon and Free Electron. British Journal of Applied Science \& Technology, 15, 1-10. https://doi.org/10.9734/BJAST/2016/25738

[29] Georgi, H. and Glashow, S.L. (1974) Unity of All Elementary Particle Forces. Physical Review Letters, 32, 438-441. https://doi.org/10.1103/PhysRevLett.32.438

[30] von Brzeski, G. and von Brzeski, V. (2018) Misconceptions of Universe Expansion, Accelerated Universe Expansion, and Their Sources. Virtual Reality of Inflationary Cosmology. Journal of Modern Physics, 9, 1326-1359.

https://doi.org/10.4236/jmp.2018.96081 